\title{
The chemistry of high-mass star formation
}

\author{
Floris F. S. van der Tak \\ Max-Planck-Institut für Radioastronomie, Auf dem Hügel 69, 53121 Bonn, Germany \\ e-mail:vdtak@mpifr-bonn.mpg.de
}

\begin{abstract}
This paper reviews the chemistry of star-forming regions, with an emphasis on the formation of high-mass stars. We first outline the basic molecular processes in dense clouds, their implementation in chemical models, and techniques to measure molecular abundances. Then, recent observational, theoretical and laboratory developments are reviewed on the subjects of hot molecular cores, cosmic-ray ionization, depletion and deuteration, and oxygen chemistry. The paper concludes with a summary of outstanding problems and future opportunities.
\end{abstract}

Keywords. astrochemistry; stars: formation

\section{Introduction: Why astrochemistry?}

The cool and dense material in the interstellar medium of galaxies plays an essential role in the life cycle of stars: it is the place where new stars are born. The only way to probe this fundamental process is through observations of molecular lines and of dust continuum at infrared and (sub-)millimeter wavelengths. The interpretation of such observations requires understanding of chemical processes for several reasons. The chemical composition of star-forming matter is very sensitive to physical parameters such as temperature, density, and ionization rate. Molecular observations thus serve as probes of physical processes such as grain growth, shocks, or cosmic-ray impact. Vice versa, observations of star formation may be aimed at a particular physical component such as the disk or the outflow by choosing lines of particular molecules. Furthermore, the chemical composition of the clouds is a strong function of time. If the temperature and density structure of a region are known, its chemical age may be estimated by comparing its observed chemical composition to model calculations. In addition, the molecular content of a region may contain information about physical conditions in its past, since the chemistry often takes significant time to respond to changes in temperature etc. Clearly, observers of star formation need at least a basic knowledge of astrochemistry.

This paper reviews the chemistry of star-forming regions, with an emphasis on regions of high-mass star formation. Section 2 gives an overview of the ingredients of star-forming matter and their interactions, both in the gas phase and in the solid phase. Section 3 discusses how these processes are incorporated into models of various physical components of star-forming regions: envelopes, hot cores, and shocks. Section 4 reviews methods to test such models: by deriving molecular abundances from spectroscopic observations at infrared and (sub-)millimeter wavelengths. Section 5 discusses recent developments in the astrochemistry of star-forming regions, grouped into four themes: hot molecular cores, cosmic-ray ionization, molecular depletion and deuteration, and oxygen chemistry.

Due to space limitations, this review is not complete, but biased toward the interests of its author. More extensive reviews of the chemical evolution of star-forming matter are given by Van Dishoeck \& Blake (1998) and Langer et al. (2000). For an overview of how to extract physical parameters such as temperatures and densities from molecular 
line observations, see Evans (1999). A recent review of the astrochemistry of low-mass star formation is given by Caselli (2005).

\section{Basics of astrochemistry}

\subsection{Ingredients of star-forming matter}

The formation of stars occurs in dense interstellar clouds with temperatures $\lesssim 100 \mathrm{~K}$ and densities $\gtrsim 10^{3} \mathrm{~cm}^{-3}$. Under these conditions, the bulk species of the gas is $\mathrm{H}_{2}$, followed by $\mathrm{He}$ at an abundance of $20 \% \dagger$. The next most abundant species is $\mathrm{CO}$, which at an abundance of $\approx 2 \times 10^{-4}$ (Van Dishoeck \& Black 1988) locks up all gas-phase carbon $\ddagger$. The remaining oxygen may be in the form of $\mathrm{H}_{2} \mathrm{O}, \mathrm{O}$ or $\mathrm{O}_{2}$, but this issue is still open (see $\S 5.4$ ). The main nitrogen carrier in dense clouds is also uncertain, due to the difficulty in observing $\mathrm{N}_{2}$ (which has no permanent dipole moment) and $\mathrm{N}$ (which has no fine structure lines). More than 100 other molecules are known $\boldsymbol{q}$, mainly through emission lines at (sub-)millimeter wavelengths.

Besides gas, star-forming matter contains solid particles or dust grains, at an abundance of $1 \%$ by mass, or about $3 \times 10^{-12}$ by number for a typical grain radius of $0.1 \mu \mathrm{m}$. The grains consist of 'cores' of amorphous carbon and silicate, covered with layers of volatile material ('ice'), which are observed through broad absorption features in the mid-infrared spectra of dense clouds and protostars. The dominant ice component is $\mathrm{H}_{2} \mathrm{O}$ at an abundance of $\sim 10^{-4}$; other known ices have abundances of $1-20 \%$ of that of $\mathrm{H}_{2} \mathrm{O}$ (see Van der Tak 2004 for a list). For all ices except CO, the solid-state abundance is much higher than can be produced in the gas phase. The data thus provide strong evidence for grain surface reactions.

\subsection{Gas phase processes}

The rate of a gas-phase reaction between species $\mathrm{A}$ and $\mathrm{B}$ is proportional to the number densities of $\mathrm{A}$ and $\mathrm{B}$ and to the rate coefficient $k$ of the reaction, which can be measured in the laboratory or calculated quantum chemically. Reactions which release energy are said to be exothermic; those which require energy to proceed are called endothermic. Reactions may have activation barriers even if they are net exothermic, because for a chemical reaction to proceed, the old chemical bond must be broken before a new one can be forged. In addition to reactions between molecules, ions and atoms, chemical reactions in interstellar clouds may involve electrons, dust grains, cosmic rays, and ultraviolet photons. The focus here is on dense clouds, defined theoretically as $n>10^{4} \mathrm{~cm}^{-3}$ or observationally as $A_{V} \gtrsim 3$. In such clouds, photoprocesses can be ignored and the main molecular processes in the gas phase are:

- Ion-molecule reactions such as $\mathrm{CO}+\mathrm{H}_{3}^{+} \rightarrow \mathrm{HCO}^{+}+\mathrm{H}_{2}$, which usually do not have activation barriers and which typically occur at the 'Langevin' rate of $\sim 10^{-9} \mathrm{~cm}^{3} \mathrm{~s}^{-1}$. These reactions dominate the chemistry of cold dark clouds. Laboratory measurements indicate that some of these reactions occur at sub-Langevin rates, for instance the $\mathrm{H}_{3}^{+}$ $+\mathrm{HD}$ reaction (Gerlich et al. 2002). However, scaling of the results from lab to space is not always straightforward and usually involves some modeling.

- Dissociative recombination reactions such as $\mathrm{HCO}^{+}+e^{-} \rightarrow \mathrm{CO}+\mathrm{H}_{2}$, which have large rate coefficients of $\sim 10^{-6} \mathrm{~cm}^{3} \mathrm{~s}^{-1}$ due to Coulomb attraction. However, due to the low electron abundance in dense clouds $\left(\sim 10^{-8}\right)$, recombination cannot compete

$\dagger$ In this paper, abundances are relative to $\mathrm{H}_{2}$ unless noted otherwise.

$\ddagger$ This paper uses words ('hydrogen') to denote elements, and symbols ('H') to denote their atomic form.

I See http://cdms.de for an up-to-date list of molecules detected in space. 
with reactions with $\mathrm{H}_{2}$ or $\mathrm{CO}$ if these are exothermic. Dissociative recombinations usually have several possible reaction products, and the 'branching ratios' between these are often uncertain. An example are recent laboratory measurements of the dissociative recombination of $\mathrm{N}_{2} \mathrm{H}^{+}$(Geppert et al. 2004).

- Neutral-neutral reactions usually have rate coefficients between $10^{-12}$ and $10^{-10}$ $\mathrm{cm}^{3} \mathrm{~s}^{-1}$, and usually (but not always) possess substantial activation barriers $(\sim 0.1 \mathrm{eV})$. These reactions are mainly important for warm, dense regions. An important example are the reactions of $\mathrm{O}$ and $\mathrm{OH}$ with $\mathrm{H}_{2}$, which drive all gas-phase oxygen into $\mathrm{H}_{2} \mathrm{O}$ at high ( $\gtrsim 300 \mathrm{~K})$ temperatures (Graff \& Dalgarno 1987).

\subsection{Solid phase processes}

Upon collision with a grain surface, atoms and molecules may be adsorbed to form ice mantles. In cold, dense pre-stellar cores $\left(n \sim 10^{4} \mathrm{~cm}^{-3}, T \sim 10 \mathrm{~K}\right)$, the evaporation time for atomic species exceeds the time to scan the entire grain surface by thermal hopping or quantum tunneling. The grain surface thus acts as a catalyst for neutral-neutral reactions at low temperatures. The reaction products are primarily saturated species (with mainly single chemical bonds), in marked contrast with gas-phase ion-molecule chemistry which tends to form species with double or multiple bonds. The composition of the ice mantle depends primarily on the atomic composition of the gas phase, with a weaker dependence on grain temperature, through differences in the volatility of atomic species (Tielens \& Hagen 1982). Recent simulations (Chang et al. 2005) and lab experiments (Hornekaer et al. 2003) show that the nature of the surface also plays an important role. The main reaction types are:

- At densities below $10^{4} \mathrm{~cm}^{-3}, \mathrm{H}$ is more abundant than $\mathrm{O}$, and hydrogenation dominates, transforming $\mathrm{O}$ into $\mathrm{H}_{2} \mathrm{O}, \mathrm{N}$ into $\mathrm{NH}_{3}, \mathrm{C}$ into $\mathrm{CH}_{4}, \mathrm{~S}$ into $\mathrm{H}_{2} \mathrm{~S}$, and $\mathrm{CO}$ into $\mathrm{H}_{2} \mathrm{CO}$ and $\mathrm{CH}_{3} \mathrm{OH}$. The observed composition of interstellar ice mantles demonstrates the dominance of this type of reaction (Gibb et al. 2000).

- At higher densities, $\mathrm{O}$ is more abundant than $\mathrm{H}$ and oxygenation is expected to become important. Support for this expectation comes from infrared observations of ubiquitous solid $\mathrm{CO}_{2}$ (Gerakines et al. 1999).

- Since the atomic $\mathrm{D} / \mathrm{H}$ ratio is orders of magnitude higher than the elemental ratio, surface chemistry may also lead to deuteration. Observational evidence for this process is provided by the high deuterium enrichment of evaporated $\mathrm{H}_{2} \mathrm{CO}$ and $\mathrm{CH}_{3} \mathrm{OH}$ in the Orion Compact Ridge (Charnley et al. 1997). However, deuterated ice species are yet to be observed (see $\S 5.3$ ).

\section{Chemical models}

The chemistry of star-forming regions is a combination of three regimes: 'dark cloud' chemistry at $T \sim 10 \mathrm{~K}$, 'hot core' chemistry at $T \sim 100 \mathrm{~K}$ and 'shock' chemistry at $T \sim 1000 \mathrm{~K}$. Figure 1 shows the location of these chemical zones.

\subsection{Envelope chemistry}

Traditional models of dark cloud chemistry follow the time evolution of an initially atomic cloud until steady state is reached after $\sim 10^{7}$ yr. Such models typically include hundreds of species and thousands of chemical reactions. If only gas-phase reactions are included, the observed abundances of some key molecules such as $\mathrm{H}_{2} \mathrm{O}$ are not well reproduced, indicating a need to include grain surface reactions (Bergin et al. 2000). Since simultaneous self-consistent treatment of gas-phase and grain-surface processes presents an enormous 


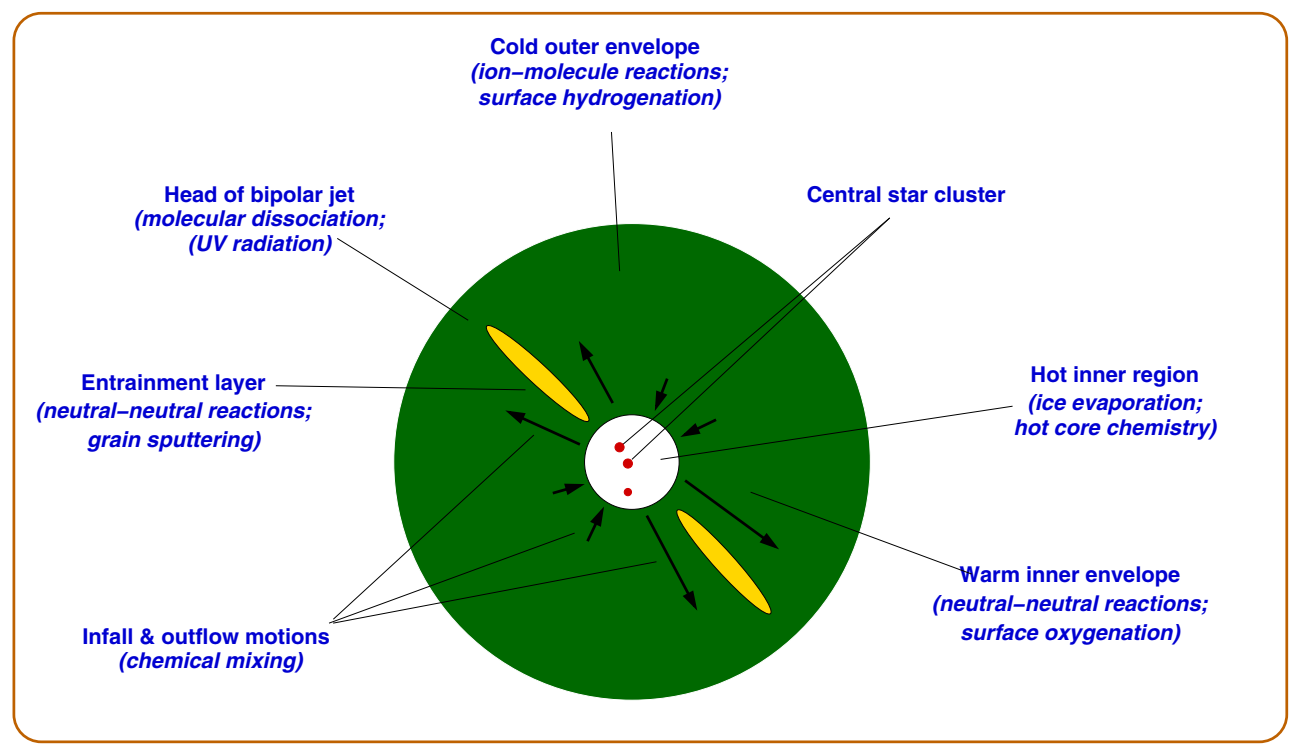

Figure 1. Schematic view of a star-forming region, with the main 'chemical zones' indicated.

computational challenge, approximate methods must be used. See Caselli (2005) for a discussion of these approximations, and also of the various possible starting conditions.

While the dark cloud models apply to regions of low temperature $(10-20 \mathrm{~K})$, evaporation of ice mantles becomes important at higher temperatures $(\gtrsim 90 \mathrm{~K})$. Models of 'hot core' chemistry follow the time evolution of warm gas after the injection of large amounts of saturated molecules which have been made on dust grain surfaces. Especially evaporated $\mathrm{CH}_{3} \mathrm{OH}$ and $\mathrm{H}_{2} \mathrm{CO}$ rapidly become protonated and react with each other to form short-lived complex organic molecules. See Van der Tak (2004) for a recent review of this process.

In recent years, dark cloud models have been combined with hot core models to describe protostellar envelopes, which have strong gradients in temperature and density. These combined models treat the chemistry as a function of both time and position. The time dependence of the models may then be used to estimate the chemical age of the region, and compare with other age estimates, for example from dynamics.

Doty and co-workers $(2002,2004)$ modeled the chemical evolution of protostellar envelopes with centrally peaked temperature and density distributions. They estimate the ages of these objects by calculating molecular column densities for various times and comparing these with values observed both in (sub-)millimeter emission and in infrared absorption. Rodgers \& Charnley (2003) and Aikawa et al. (2005) take this approach one step further and consider the effect of the kinematics of such envelopes on their chemical evolution. Very precise observations of many molecular species are needed to test these models, which have many free parameters. For most species, agreement to factors of $2-3$ can be obtained for reasonable ages, but some species deviate much more, indicating problems with either the initial conditions of the models or with the assumed reaction rates.

\subsection{Shock chemistry}

The third type of chemistry relevant for star-forming regions is that of interstellar shocks, which occur when powerful jets from young stars collide with ambient material. The chemistry of the shocked gas depends on the shock velocity. Shocks with velocities 
$\lesssim 40 \mathrm{~km} \mathrm{~s}^{-1}$ heat the ambient gas to a few $1000 \mathrm{~K}$ and result in copious $\mathrm{H}_{2} \mathrm{O}$, SO and $\mathrm{SO}_{2}$ production in neutral-neutral reactions (Kaufman \& Neufeld 1996). In contrast, faster shocks will dissociate and ionize the gas, heat it to $10^{5} \mathrm{~K}$ and produce copious ultraviolet radiation; molecules then re-form slowly in the warm postshock gas and a largely molecule-free zone is created (Hollenbach \& McKee 1989). Such fast shocks probably occur where the ambient gas takes a direct hit from a protostellar jet, while slower shocks take place along the sides (Fig. 1).

The grains stay cool in either type of shock, but their charge causes them to interact with the gas. In particular, slow shocks in magnetized regions cause ion-neutral drift, and thus to collisions of grains with $\mathrm{H}_{2}$ molecules. These collisions erode the grains, liberating volatile material from their mantles and refractory material from their cores. The enhanced abundances of $\mathrm{CH}_{3} \mathrm{OH}$ and $\mathrm{SiO}$ observed towards protostellar outflows are thought to originate in such 'sputtering' (Bachiller et al. 2001). The recent first detections of the $\mathrm{FeO}$ and $\mathrm{SiN}$ molecules (Walmsley et al. 2002; Schilke et al. 2003) form additional tests of this scenario.

Recently, models have been developed which combine hot core chemistry with shock chemistry, although observational tests are still inconclusive (Hatchell \& Viti 2002). Models which incorporate all three regimes (dark clouds, hot cores, and shocks) do not exist yet, but would be valuable to describe star-forming regions. One approach would be to develop axisymmetric models, where envelope chemistry proceeds along the equator, and shock chemistry close to the axis.

\section{Measuring molecular abundances}

\subsection{Observational techniques}

The observational part of astrochemistry consists of spectroscopy of molecular rotational lines at (sub-)millimeter wavelengths and of ro-vibrational lines at near- and mid-infrared wavelengths. Either type of line may appear in emission or in absorption, depending on geometry. However, since molecular vibrational states lie at much higher energies $(\sim 1000 \mathrm{~K})$ than rotational states $(\sim 10-100 \mathrm{~K})$, the excitation of (sub-)millimeter rotational lines occurs over much larger regions than that of mid-infrared ro-vibrational lines. In addition, absorption measurements require a background source, of which many more are available in the mid-infrared than in the (sub-)millimeter. Therefore, mid-infrared lines usually appear in absorption and (sub-)millimeter lines in emission.

The advantage of absorption spectroscopy is that the angular resolution is not limited by the size of the telescope, but by the (usually very small) size of the background continuum source. On the other hand, if the lines appear in emission, they can be mapped, either by scanning the telescope or by using array receivers.

In the mid-infrared, the molecular excitation can be measured accurately since many ro-vibrational lines occur close together in wavelength. Furthermore, molecules without permanent dipole moments $\left(\mathrm{C}_{2} \mathrm{H}_{2}, \mathrm{CH}_{4}, \ldots\right)$ can only be probed in the mid-infrared, as is the case for solid-state features. On the other hand, the advantage of (sub-)millimeter spectra is that even the narrowest spectral features can be velocity-resolved. Furthermore, rotational lines have a much higher sensitivity (down to abundances of $\sim 10^{-12}$ ) than ro-vibrational lines. Clearly, the infrared and (sub-)millimeter techniques are complementary, and both are needed for a complete picture of the chemistry in star-forming regions (e.g., Boonman et al. 2003). 


\subsection{Radiative transfer techniques}

Traditionally, molecular abundances are estimated as the ratio of the molecular column density $N_{X}$ and the $\mathrm{H}_{2}$ column density $N_{H}$. The conversion from line strength to column density requires estimates of the molecular excitation and the line optical depth. If several lines of the molecule have been observed, the excitation may be estimated from the relative strengths of these lines. If only one line has been observed, the excitation must be estimated from observations of other species, or from theoretical considerations. The optical depth of an absorption line follows from the line to continuum ratio if the profile is resolved; otherwise, the 'curve of growth' method may be applied, assuming an intrinsic line width. For emission lines, the optical depth is usually estimated from the line strength, assuming that the emission fills the telescope beam. These estimates may be done analytically, or numerically using the Large Velocity Gradient (LVG) or similar 'local' approximations. In the same way, $N_{H}$ is obtained from observations of CO isotopic lines or dust continuum, and the 'average abundance' $N_{X} / N_{H}$ may be compared with chemical models.

The traditional procedure is appropriate for large-scale molecular clouds which are homogeneous on the scales probed by single-dish telescopes, although the determination of the appropriate $N_{H}$ often introduces considerable uncertainty. In star-forming regions with strong temperature and density gradients and chemical differentiation, the procedure may be used as a first guess if little data are available. However, deviations from the assumptions of uniform excitation and uniform abundance along the line of sight are considerable, and more sophisticated methods are called for. In recent years, the use of Monte Carlo (MC) or Accelerated Lambda Iteration (ALI) programs has become common. Such programs take temperature, density and velocity gradients within the source into account, and also open the possibility to test scenarios where molecular abundances vary with location. If enough observations of a molecule are available, an abundance profile along the line of sight can be derived, rather than a mere average. An outline of the procedure to extract such abundance profiles from multi-line observations is given by Doty et al. (2004). The first results from such models include the finding of increased abundances ('jumps') of $\mathrm{CH}_{3} \mathrm{OH}, \mathrm{H}_{2} \mathrm{CO}$, $\mathrm{SO}$ and $\mathrm{SO}_{2}$ in the warm $(>100 \mathrm{~K}$ ) gas close to young stars (Van der Tak et al. 2000, 2003; Schöier et al. 2002) which are likely due to the evaporation of icy grain mantles. More recently, abundance decreases ('drops') of CO and $\mathrm{HCO}^{+}$have been found in the cold outer parts of low-mass protostellar envelopes due to freeze-out of CO onto the grains (Jørgensen et al. 2004b). Direct observational evidence for such differentiation usually requires high angular resolution $\left(\lesssim 1^{\prime \prime}\right)$ as provided by interferometers. The images of the 'double hot core' in the warm gas close to one young low-mass star (Bottinelli et al. 2004; Kuan et al. 2004) and of chemical changes along the outflow of another (Jørgensen et al. 2004a) provide two recent examples.

Both LVG and MC/ALI programs need spectroscopic and collisional input data for the molecule that has been observed. Until recently, such data were scattered all over the physical, chemical and astronomical literature in many different formats. Data for the most commonly observed molecules have now been collected into a database (Schöier et al. 2005). The associated website $\dagger$ contains data files for many molecular and atomic species, and also a simple LVG-type line strength calculator. 


\section{Recent developments}

\subsection{Hot core chemistry}

Hot molecular cores are regions close to young stars, where ice mantles evaporate off dust grains and high-temperature gas-phase chemistry creates short-lived complex organic species. The result are very rich (sub-)millimeter spectra, for instance as observed at $800 \mathrm{GHz}$ toward Orion-KL (Comito et al. 2005). This work is a nice illustration of modern observing capabilities, but also of the difficulties in assigning molecular lines in very crowded spectra. Indeed, the claimed detection of interstellar glycine remains disputed (Snyder et al. 2005) because of ambiguities in the line identification.

The short time scale of the hot core phenomenon $\left(\sim 10^{4} \mathrm{yr}\right)$ makes it a possible 'chemical clock', but this application requires understanding which species are formed on the grain surface and/or in the gas phase. Recent years have seen progress toward this goal from several directions, both observational and theoretical. Ground-based high-resolution mid-infrared spectroscopy has provided the first detection of solid ${ }^{13} \mathrm{CO}$ towards one highmass protostar (Boogert et al. 2002). The differences in the absorption profiles between ${ }^{12} \mathrm{CO}$ and ${ }^{13} \mathrm{CO}$ contain information about the structure of the ice matrix and its evolution (see also Palumbo, these proceedings). Along the same line of sight, variations of the $\mathrm{CH}_{4}$ abundance were found (Boogert et al. 2004a), which suggest that $\mathrm{CH}_{4}$ is made on grains but quickly destroyed after evaporation into the gas phase. Interestingly, the spectrum also shows possible absorption by solid $\mathrm{C}_{2} \mathrm{H}_{6}$ and $\mathrm{C}_{2} \mathrm{H}_{5} \mathrm{OH}$ ! The first inventories of solid state features toward low-mass protostars have been made with the Spitzer space observatory (Boogert et al. 2004b). The large $\mathrm{CO}_{2} / \mathrm{CH}_{3} \mathrm{OH}$ ratios suggests that the ices were formed under dense conditions, while the band profiles suggest that thermal processing of the ice is less important than for high-mass stars. Mapping of solid-state features in the Serpens star-forming region (Pontoppidan et al. 2004) suggests that the ice formation efficiency is strongly density dependent.

The hot core phenomenon also remains the subject of theoretical investigation, especially the formation of complex species in the gas phase. Quantitative understanding of these processes is still lacking, as the recent example of methyl formate shows (Horn et al. 2004). Another recent result is that the isomerization of $\mathrm{H}_{2} \mathrm{CO}$ and $\mathrm{CH}_{3} \mathrm{OH}$, in particular the shifting of a $\mathrm{D}$ atom from one side of the molecule to another, appears inefficient (Osamura et al. 2004).

\subsection{Cosmic-ray ionization}

The ionization fraction of molecular clouds determines the importance of magnetic fields for their dynamics, and sets the time scale of ion-molecule chemistry. In star-forming regions, which are shielded from ultraviolet radiation, the main source of ionization is by cosmic rays. The bulk of the ionizations convert $\mathrm{H}_{2}$ into $\mathrm{H}_{3}^{+}\left(\right.$via $\left.\mathrm{H}_{2}{ }^{+}\right)$, but a small fraction $(\approx 3 \%)$ of cosmic rays produce $\mathrm{He}^{+}$. The He ions are important because they are the only ones capable of breaking strong chemical bonds such as in $\mathrm{CO}$ or $\mathrm{N}_{2}$. Observations of $\mathrm{H}_{3}^{+}$absorption and $\mathrm{H}^{13} \mathrm{CO}^{+}$emission lines toward seven young high-mass stars indicate a cosmic-ray ionization rate of $\zeta_{\mathrm{CR}} \approx 3 \times 10^{-17} \mathrm{~s}^{-1}$ (Van der Tak \& van Dishoeck 2000). Very close to high-mass stars, photo-ionization plays an additional role, as the detection of $\mathrm{CO}^{+}$and $\mathrm{SO}^{+}$toward AFGL 2591 testifies (Stäuber et al. 2005).

Recent observations indicate significant spatial variations of $\zeta_{\mathrm{CR}}$. Spectroscopy of $\mathrm{H}_{3}^{+}$ at $3.5 \mu \mathrm{m}$ toward a local diffuse cloud (McCall et al. 2003) indicates a local enhancement of $\zeta_{\mathrm{CR}}$ relative to the above value by about a factor of 10, taking the distribution of species along the line of sight into account (Le Petit et al. 2004). On the other hand, observations of emission lines of $\mathrm{DCO}^{+}$and other ions toward highly shielded pre-stellar cores indicate 
a decrease of $\zeta_{\mathrm{CR}}$ by a factor of 10 (Caselli et al. 2002), although depletion may influence this result. The apparent scaling of $\zeta_{\mathrm{CR}}$ with density may be related to magnetic fields. Denser regions have stronger magnetic fields (Crutcher, these proceedings) and are more able to deflect impinging cosmic rays. Models by Padoan \& Scalo (2005) predict a scaling of $\zeta_{\mathrm{CR}}$ with density as a result of cosmic-ray confinement by self-generated MHD waves. More observations are needed to test these and other models.

\subsection{Depletion and Deuteration}

Another area where astrochemistry is useful are the extreme physical conditions implied by observations of very high molecular $\mathrm{D} / \mathrm{H}$ ratios in pre-stellar cores and young protostellar envelopes. Observations have confirmed the theoretical expectation that the strong deuteration is linked to the depletion of $\mathrm{CO}$ from the gas phase by freeze-out on dust grains (Bacmann et al. 2003). Current chemical models use depletion by factors of up to $\sim 10$ to explain observations of single and double deuteration (e.g., Roberts \& Millar 2000), but recent observations of triply deuterated molecules (Lis et al. 2002; Van der Tak et al. 2002; Parise et al. 2004; Roueff et al. 2005) and of highly abundant $\mathrm{H}_{2} \mathrm{D}^{+}$ and $\mathrm{D}_{2} \mathrm{H}^{+}$(Caselli et al. 2003; Vastel et al. 2004) have shown that under extreme conditions, essentially all (>99\%) of molecules containing nuclei heavier than He are frozen out. Such conditions occur at the centers of very dense pre-stellar cores on the verge of forming stars. Very soon after stars begin to form, the heavy elements re-appear in the gas phase and the level of deuteration goes down. The near-absence of heavy elements from the gas phase has far-reaching consequences for the chemistry, as shown in a series of papers by the 'Deuteronomy' team (Walmsley et al. 2004; Flower et al. 2005). The results indicate that the abundances of $\mathrm{H}_{2} \mathrm{D}^{+}$and $\mathrm{D}_{2} \mathrm{H}^{+}$may be used to constrain the grain size distribution.

The role of surface chemistry in enhancing molecular $\mathrm{D} / \mathrm{H}$ ratios is uncertain, since to date no D-bearing species are observed in the solid state (Dartois et al. 2003; Parise et al. 2003). Observed D/H enhancements around young high-mass stars are $\sim 100 \times$ lower than around low-mass stars, but still much higher than expected from the observed gas temperature (Gensheimer et al. 1996). In this case the $\mathrm{D} / \mathrm{H}$ ratio is a 'fossil' from an earlier, colder phase, such as the recently discovered infrared dark cloud cores.

\subsection{Oxygen chemistry}

As mentioned in $\S 2.1$, the major oxygen carrier in dense interstellar clouds is not well known. Two recent space missions (SWAS and ODIN) have measured (sub-)millimeter lines of $\mathrm{H}_{2} \mathrm{O}$ and $\mathrm{O}_{2}$ to answer this question. The derived $\mathrm{H}_{2} \mathrm{O}$ abundances range from $\sim 10^{-4}$ in warm $(\gtrsim 300 \mathrm{~K})$ gas to $\sim 10^{-8}$ in cold $(\$ 30 \mathrm{~K})$ clouds (Snell et al. 2000$)$. The $\mathrm{O}_{2}$ molecule remains elusive, with the current best limit on its abundance of $<10^{-7}$ (Pagani et al. 2003). These observations imply that in dense interstellar clouds, $\mathrm{O}_{2}$ is never a major oxygen carrier nor a major gas coolant, and $\mathrm{H}_{2} \mathrm{O}$ only in localized warm regions. The large variations in $\mathrm{H}_{2} \mathrm{O}$ abundance and the absence of $\mathrm{O}_{2}$ can be explained by freezeout of oxygen onto dust grains $(\$ 100 \mathrm{~K})$, ice evaporation $(\gtrsim 100 \mathrm{~K})$, and neutral-neutral reactions in the gas phase $(\gtrsim 250 \mathrm{~K})$.

Several questions remain unanswered in this area. The $\mathrm{H}_{2} \mathrm{O}$ abundances are based on a single transition, and the excitation had to be estimated from external data. The abundances also only refer to ortho- $\mathrm{H}_{2} \mathrm{O}$, while most $\mathrm{H}_{2} \mathrm{O}$ may be in para form in cold clouds. Third, $\mathrm{H}_{2} \mathrm{O}$ remains undetected in several classes of objects, notably low-mass star-forming cores. Finally, the link between the high $\mathrm{H}_{2} \mathrm{O}$ abundances on small scales and the low values on large scales is still poorly understood (e.g., Boonman et al. 2003). The HIFI spectrometer and PACS camera onboard the Herschel space observatory will allow 
key progress on these questions. These instruments will carry out multi-line observations of $\mathrm{H}_{2} \mathrm{O}$ in $\approx 10-20^{\prime \prime}$ beams. For further discussion see Walmsley \& van der Tak (2005).

\section{Conclusions and future opportunities}

As stated in $\S 1$, the two uses of astrochemistry for star formation studies are: (1) understanding which molecule traces which physical component; (2) estimate the chemical ages of star-forming regions. The impression of this reviewer is that the current understanding of astrochemistry is good enough for the first goal, but not for the second. However, this situation may well change in the next few years, when many new facilities come on-line.

New mid-infrared observations, both from the ground and with Spitzer and ultimately JWST, will be useful to make deeper inventories of the composition of icy grain mantles than currently possible. In particular, the main nitrogen and sulphur carriers are waiting to be identified, as are deuterated constituents. Many species known from (sub-)millimeter observations are still awaiting detection by gas-phase spectroscopy in the mid-infrared. The combination of these techniques will be very powerful in constraining the location of these molecules (for example $\mathrm{CH}_{3} \mathrm{OH}$ ) and thus in building up a 3D picture of the chemical structure of star-forming regions.

The launch of the Herschel space observatory in 2007 will allow spectral line surveys at Terahertz frequencies with the HIFI instrument. These observations will considerably extend our knowledge of the gas-phase composition of star-forming regions, particularly for light species such as hydrides. As discussed in $\S 5.4$, the origin and evolution of $\mathrm{H}_{2} \mathrm{O}$ in star-forming regions is another major goal of Herschel.

Last but not least, the advent of high angular resolution at (sub-)millimeter wavelengths with ALMA will allow us to study chemical structure on small scales. For example, this telescope will be able to resolve regions with enhanced abundances of, e.g., $\mathrm{CH}_{3} \mathrm{OH}$ and $\mathrm{SO}_{2}$ in the inner envelopes of young high-mass stars. Also of great interest is the chemistry of infrared dark cloud cores which likely represent the initial conditions of high-mass star formation. Clearly, the next 5-10 years will be an exciting time for the students of astrochemistry.

\section{Acknowledgements}

The author thanks Malcolm Walmsley and Ted Bergin for useful discussions, and the organizers of the symposium for their invitation to give this review.

\section{References}

Aikawa, Y., Herbst, E., Roberts, H., \& Caselli, P. 2005, ApJ, 620, 330

Bachiller, R., Pérez Gutiérrez, M., Kumar, M. S. N., \& Tafalla, M. 2001, A\&A, 372, 899

Bacmann, A., Lefloch, B., Ceccarelli, C., et al. 2003, ApJ, 585, L55

Bergin, E. A., Melnick, G. J., Stauffer, J. R., et al. 2000, ApJ, 539, L129

Boogert, A. C. A., Blake, G. A., \& Öberg, K. 2004a, ApJ, 615, 344

Boogert, A. C. A., Blake, G. A., \& Tielens, A. G. G. M. 2002, ApJ, 577, 271

Boogert, A. C. A., Pontoppidan, K. M., Lahuis, F., et al. 2004b, ApJS, 154, 359

Boonman, A. M. S., Doty, S. D., van Dishoeck, E. F., et al. 2003, A\&A, 406, 937

Bottinelli, S., Ceccarelli, C., Neri, R., et al. 2004, ApJ, 617, L69

Caselli, P. 2005, in astro-ph, 0504298

Caselli, P., van der Tak, F. F. S., Ceccarelli, C., \& Bacmann, A. 2003, A\&A, 403, L37

Caselli, P., Walmsley, C. M., Zucconi, A., et al. 2002, ApJ, 565, 344

Chang, Q., Cuppen, H. M., \& Herbst, E. 2005, A\&A, 434, 599 
Charnley, S. B., Tielens, A. G. G. M., \& Rodgers, S. D. 1997, ApJ, 482, L203

Comito, C., Schilke, P., Phillips, T. G., et al. 2005, ApJS, 156, 127

Dartois, E., Thi, W.-F., Geballe, T. R., et al. 2003, A\&A, 399, 1009

Doty, S., van Dishoeck, E., van der Tak, F., \& Boonman, A. 2002, A\&A, 389, 446

Doty, S. D., Schöier, F. L., \& van Dishoeck, E. F. 2004, A\&A, 418, 1021

Evans, N. J. 1999, ARA\&A, 37, 311

Flower, D. R., Pineau des Forêts, G., \& Walmsley, C. M. 2005, A\&A, in press

Gensheimer, P. D., Mauersberger, R., \& Wilson, T. L. 1996, A\&A, 314, 281

Geppert, W. D., Thomas, R., Semaniak, J., et al. 2004, ApJ, 609, 459

Gerakines, P. A., Whittet, D. C. B., Ehrenfreund, P., et al. 1999, ApJ, 522, 357

Gerlich, D., Herbst, E., \& Roueff, E. 2002, Pl. Sp. Sc. , 50, 1275

Gibb, E. L., Whittet, D. C. B., Schutte, W. A., et al. 2000, ApJ, 536, 347

Graff, M. M. \& Dalgarno, A. 1987, ApJ, 317, 432

Hatchell, J. \& Viti, S. 2002, A\&A, 381, L33

Hollenbach, D. \& McKee, C. F. 1989, ApJ, 342, 306

Horn, A., Møllendal, H., Sekiguchi, O., et al. 2004, ApJ, 611, 605

Hornekaer, L., Baurichter, A., Petrunin, V., Field, D., \& Luntz, A. 2003, Science, 302, 1943

Jørgensen, J. K., Hogerheijde, M. R., Blake, G. A., et al. 2004a, A\&A, 415, 1021

Jørgensen, J. K., Schöier, F. L., \& van Dishoeck, E. F. 2004b, A\&A, 416, 603

Kaufman, M. J. \& Neufeld, D. A. 1996, ApJ, 456, 611

Kuan, Y., Huang, H., Charnley, S. B., et al. 2004, ApJ, 616, L27

Langer, W. D., van Dishoeck, E. F., Bergin, E. A., et al. 2000, Protostars and Planets IV, 29

Le Petit, F., Roueff, E., \& Herbst, E. 2004, A\&A, 417, 993

Lis, D. C., Roueff, E., Gerin, M., et al. 2002, ApJ, 571, L55

McCall, B. J., Huneycutt, A. J., Saykally, R. J., et al. 2003, Nature, 422, 500

Osamura, Y., Roberts, H., \& Herbst, E. 2004, A\&A, 421, 1101

Padoan, P. \& Scalo, J. 2005, ApJ, 624, L97

Pagani, L., Olofsson, A. O. H., Bergman, P., et al. 2003, A\&A, 402, L77

Parise, B., Castets, A., Herbst, E., et al. 2004, A\&A, 416, 159

Parise, B., Simon, T., Caux, E., et al. 2003, A\&A, 410, 897

Pontoppidan, K. M., van Dishoeck, E. F., \& Dartois, E. 2004, A\&A, 426, 925

Roberts, H. \& Millar, T. J. 2000, A\&A, 361, 388

Rodgers, S. D. \& Charnley, S. B. 2003, ApJ, 585, 355

Roueff, E., Lis, D. C., van der Tak, F. F. S., Gerin, M., \& Goldsmith, P. F. 2005, A\&A, in press

Schöier, F. L., Jørgensen, J. K., van Dishoeck, E. F., \& Blake, G. A. 2002, A\&A, 390, 1001

Schöier, F. L., van der Tak, F. F. S., van Dishoeck, E. F., \& Black, J. H. 2005, A\&A, 432, 369

Schilke, P., Leurini, S., Menten, K. M., \& Alcolea, J. 2003, A\&A, 412, L15

Snell, R. L., Howe, J. E., Ashby, M. L. N., et al. 2000, ApJ, 539, L101

Snyder, L. E., Lovas, F. J., Hollis, J. M., et al. 2005, ApJ, 619, 914

Stäuber, P., Benz, A. O., Doty, S. D., \& van Dishoeck, E. F. 2005, in The Dusty and Molecular Universe: A Prelude to Herschel and ALMA, 413-414

Tielens, A. G. G. M. \& Hagen, W. 1982, A\&A, 114, 245

Van der Tak, F., Boonman, A., Braakman, R., \& van Dishoeck, E. 2003, A\&A, 412, 133

Van der Tak, F. F. S. 2004, in IAU Symposium 221, 59

Van der Tak, F. F. S., Schilke, P., Müller, H. S. P., et al. 2002, A\&A, 388, L53

Van der Tak, F. F. S. \& van Dishoeck, E. F. 2000, A\&A, 358, L79

Van der Tak, F. F. S., van Dishoeck, E. F., \& Caselli, P. 2000, A\&A, 361, 327

Van Dishoeck, E. F. \& Black, J. H. 1988, ApJ, 334, 771

Van Dishoeck, E. F. \& Blake, G. A. 1998, ARA\&A, 36, 317

Vastel, C., Phillips, T. G., \& Yoshida, H. 2004, ApJ, 606, L127

Walmsley, C. M., Bachiller, R., Forêts, G. P. d., \& Schilke, P. 2002, ApJ, 566, L109

Walmsley, C. M., Flower, D. R., \& Pineau des Forêts, G. 2004, A\&A, 418, 1035

Walmsley, C. M. \& van der Tak, F. F. S. 2005, in The Dusty and Molecular Universe: A Prelude to Herschel and ALMA, 55-60 